\title{
A Sensitive Method of Non-Radioactive In Situ Hybridization for mRNA Localization within Human Renal Biopsy Specimens: Use of Digoxigenin Labeled Oligonucleotides
}

\author{
Masanobu Mryazaki, David J. Nikolic-Paterson*, Masayuki Endoh, Yasuo Nomoto, \\ Hideto SAKAI, Robert C. AtKINs* and Takehiko KoII**
}

\begin{abstract}
A sensitive method of non-radioactive in situ hybridization using digoxigenin-labeled oligonucleotides is described for the detection of mRNA within human renal biopsy specimens. Although non-radioactive in situ hybridization typically has the drawback of low sensitivity, we increased the sensitivity of this method, providing a practical alternative to the use of radiolabelled probes. The four main points are: 1) assessment of the efficiency of labeling, 2) optimization of the probe concentration for hybridization, 3) requirement of deproteinization of tissues with $\mathrm{HCl}$ and proteinase $K$, and 4) the use of a four-layer immunoperoxidase staining system. This technique was found to clearly localize individual mRNA positive cells within cryostat tissue sections. A variety of controls including sense probes, excess unlabeled anti-sense probes, and RNase-treatment demonstrated the specificity of the technique. This improved method is a powerful technique for detecting mRNA within human renal tissue and will be most useful in the study of gene expression in the pathogenesis of renal diseases.
\end{abstract}

(Internal Medicine 33: 87-91, 1994)

Key words: in situ hybridization, digoxigenin, renal biopsy, IL-6

\section{Introduction}

There are three methods for the detection of mRNA in tissues: Northern blotting, polymerase chain reaction (PCR), and in situ hybridization. When there is adequate tissue, Northern blotting provides a quantitative measurement of mRNA content. However, most human specimens yield too little RNA to allow Northern blotting, and so amplification by PCR is a powerful technique to detect the presence of mRNA. PCR is widely used in clinical medicine for the detection of viruses and bacteria, as well as in genetic medicine $(1,2)$. However, neither Northern blotting or PCR can localize the cells expressing mRNA within tissue specimens. To do this, the technique of in situ hybridization is required. There are two basic ways in which nucleic acid probes are labeled for in situ hybridization; radioactive isotopes and non-radioactive haptens. The former are detected by liquid emulsion autoradiography and the latter by immunohistochemistry. Non-radioactive probes have the advantages of high resolution, stability, and short development times. However, the main drawback of non-radioactive probes has been their relatively low sensitivity. Recently, some improvement in the sensitivity of non-radioactive probes has been reported (3). Based on these observations, we have developed a sensitive method of non-radioactive in situ hybridization using digoxigenin with the aim of detecting specific cytokine mRNA within human renal biopsy specimens. The key points of this method are described.

\section{Material and Methods}

\section{In situ hybridization}

Fresh kidney biopsy specimens obtained from patients with IgA nephropathy were embedded in O.C.T. compounds (Tissue-Tek ${ }^{\circledR}$, Miles Inc., Elkhart, IN, USA) and stored at $-70^{\circ} \mathrm{C}$ until experimental use. These specimens were cut to a thickness of 6 to $8 \mu \mathrm{m}$ and adhered to gelatin-coated slide glass. They were fixed with $4 \%$ paraformaldehyde (PFA) in phosphatebuffered saline (PBS) on ice for 15 minutes and washed three times in PBS. Sections were deproteinized by treatment with $0.2 \mathrm{~N} \mathrm{HCl}$ and digested with $1 \mu \mathrm{g} / \mathrm{ml}$ of proteinase K (Sigma P-

\footnotetext{
From the Department of Nephrology, Tokai University School of Medicine, Isehara, *Department of Nephrology, Monash Medical Center, Clayton, Australia and **Department of Anatomy, Nagasaki University School of Medicine, Nagasaki

Received for publication April 28, 1993; Accepted for publication December 20, 1993

Reprint requests should be addressed to Dr. Masanobu Miyazaki, the Second Department of Internal Medicine, Nagasaki University, School of Medicine, 1-7-1, Sakamoto, Nagasaki 852
} 
4914, St. Louis, U.S.A.) in PBS at $37^{\circ} \mathrm{C}$. Optimal incubation time for each step was determined according to the thickness of the section. Prehybridization was performed at $37^{\circ} \mathrm{C}$ for 60 minutes in a solution containing $4 \times \mathrm{SSC}, 5 \times$ Denhardt's solution, $0.2 \mathrm{mg} / \mathrm{ml}$ of salmon testis DNA (Sigma D-7656), $0.2 \mathrm{mg}$ / $\mathrm{ml}$ of yeast tRNA (Sigma R-8508) and $50 \mathrm{mM}$ sodium phosphate $\mathrm{pH}$ 8.0. Sections were drained and hybridized with $1 \mathrm{ng} /$ $\mu \mathrm{l}$ of digoxigenin (DIG)-labeled oligonucleotide probe in prehybridization buffer at $37^{\circ} \mathrm{C}$ overnight. Optimal concentration of probes had been determined by several preliminary studies. The sections were then washed with $2 \times$ and $1 \times$ salinesodium citrate buffer ( $\mathrm{SSC}$ ) two times at room temperature for 30 minutes. DIG-labeled probes were visualized by immunocytochemical staining. In order to preserve mRNA in tissues, all solutions were treated with diethlpyrocarbonate (DEPC) and autoclaved prior to use.

\section{Immunocytochemical staining}

Immunoperoxidase staining was performed as follows. After washing with hybridization buffer, sections were preincubated with blocking solution containing $20 \%$ normal swine serum (NSS, Dakopatts X901, Denmark), 5\% fetal calf serum (FCS, Gibco BRL, Gaithersburg, MD, U.S.A.) and $5 \%$ bovine serum albumin (BSA, Sigma) in buffer 1 (100 mM NaCl, $150 \mathrm{mM}$ Tris- $\mathrm{HCl}, \mathrm{pH} 7.5)$ for $30 \mathrm{~min}$ to prevent non-specific binding. After draining, mouse monoclonal anti-DIG antibody (Boehringer Mannheim Biochemica 1333062, West Germany) was overlaid on the section at $4^{\circ} \mathrm{C}$ overnight. After washing three times with buffer 1 , the sections were dehydrated through graded ethanol and incubated in methanol with $0.3 \% \mathrm{H}_{2} \mathrm{O}_{2}$ at room temperature for 20 minutes to block endogenous peroxidase and washed three times in buffer 2 [buffer 1 with $0.02 \%$ Tween 20 (Wako 163-11512, Tokyo, Japan)]. The sections were then incubated sequentially with horse raddish peroxidase (HRP)conjugated rabbit anti-mouse antibody (Dakopatts P260), HRPconjugated swine anti-rabbit antibody (Dakopatts P399) and complexes of peroxidase rabbit polyclonal anti-peroxidase (Dakopatts Z113) with three washings in buffer 2 between each step. Color was developed by reaction with $0.6 \mathrm{mg} / \mathrm{ml}$ of diaminobenzidine tetrahydrochloride in $0.05 \mathrm{M}$ Tris- $\mathrm{HCl}, \mathrm{pH}$ 7.6, plus $0.03 \% \mathrm{H}_{2} \mathrm{O}_{2}$. Sections were counterstained briefly with hematoxylin, rinsed, dehydrated through graded ethanol, cleared in xylene and mounted. Two and three layer staining methods were compared with the four-layer method to assess sensitivity.

To evaluate the specificity of the technique, RNase treatment, a study with a sense probe and a competitive study using large amounts of unlabeled probes were performed as follows. Sections were incubated with $1 \mathrm{mg} / \mathrm{ml}$ RNase (Boehringer Mannheim Biochemica 109169) at $37^{\circ} \mathrm{C}$ for 30 minutes after proteinase $\mathrm{K}$ digestion and prehybridized and hybridized as described above. A sense probe which was complementary to the antisense probe was added to the section in place of the antisense probe. A competitive study was performed by addition of 100-fold the amount of the same or unrelated unlabeled oligonucleotides to the hybridization buffer.
Staining of stimulated peripheral blood mononuclear cells $(P B M C)$ as positive controls

LPS-stimulated PBMC served as the positive physiological control of certain types of cytokine. Heparinized venous blood was collected from healthy adults, and mononuclear cells were separated by Ficoll centrifugation gradient. Ten thousand cells were incubated with or without the addition of $1 \mathrm{ng} / \mathrm{ml}$ lipopolysaccharide (LPS, purchased from Sigma) for $6 \mathrm{hrs}$ at $37^{\circ} \mathrm{C}$. After harvesting and washing, cells were suspended in $1 \%$ BSA in PBS and adhered to a slide glass using cytospin (Shandon Inc., Pittsburgh, PA, USA). These glass slides were stored at $-70^{\circ} \mathrm{C}$ until in situ hybridization.

\section{Probes and labeling}

The 20 mer oligonucleotide probe was designed based on the sequence of IL-6 (4) and B-actin (5) by a computer and synthesized by a DNA synthesizer (380B DNA synthesizer, Applied Biosystem). The sequence of IL-6 probe was 5' GCTTGTTCCTCACTACTCTC 3'. For B-actin, the probe sequence was 5' AGCCTGGATAGCAACGTACA 3'. These probes were RNA specific and non-reactive with DNA. The specificity of the oligonucleotides was examined by Southern blotting to detect binding to the original cDNA probe. One hundred picomole of the oligonucleotide probe was labeled using a DIG oligonucleotide tailing kit according to the standard protocol (Boehringer 1417 231). Free DIG was removed by ethanol precipitation and dissolved in DEPC-treated water. To assess labeling efficacy, the labeled probe was diluted several times from $1 \mathrm{ng} / \mu \mathrm{l}$ to $1 \mathrm{pg} / \mu \mathrm{l}$ and spotted on a nylon membrane (Hybond-N, Amersham, Buckinghamshire, England). The membrane was stained using the same four-layer immunoperoxidase technique described above in a sealed polyethylene bag.

\section{Results}

The most critical component of this in situ hybridization technique was the immunoperoxidase detection system. Comparisons between 2-, 3- and 4-layer immunoperoxidase staining were made. It was found that the two-layer system gave very weak staining and it could not be clearly recognized as positive. The three-layer system improved the signal, but by far the best result was obtained with the four-layer staining condition. The DIG-labeled IL-6 probe was titrated on $6 \mu \mathrm{m}$ sections using the 4-layer detection system. A concentration of $1 \mathrm{ng} / \mu \mathrm{l}$ of probe was found to produce the optimal signal with the least background. It was found that deproteinization of tissue sections with $\mathrm{HCl}$ and proteinase $\mathrm{K}$ significantly improved the hybridization signal. However, deproteinization did cause a loss of morphology. A ten-minute incubation with $1 \mu \mathrm{g} / \mathrm{ml}$ proteinase $\mathrm{K}$ gave the best signal with the least deterioration of morphology. The use of sections more than $8 \mu \mathrm{m}$ thick caused difficulties in localization of the signal due to the presence of overlapping cells within the section.

In situ hybridization using a DIG-labeled IL-6 oligonucleotide probe on a renal biopsy specimen from a patient with $\operatorname{IgA}$ 

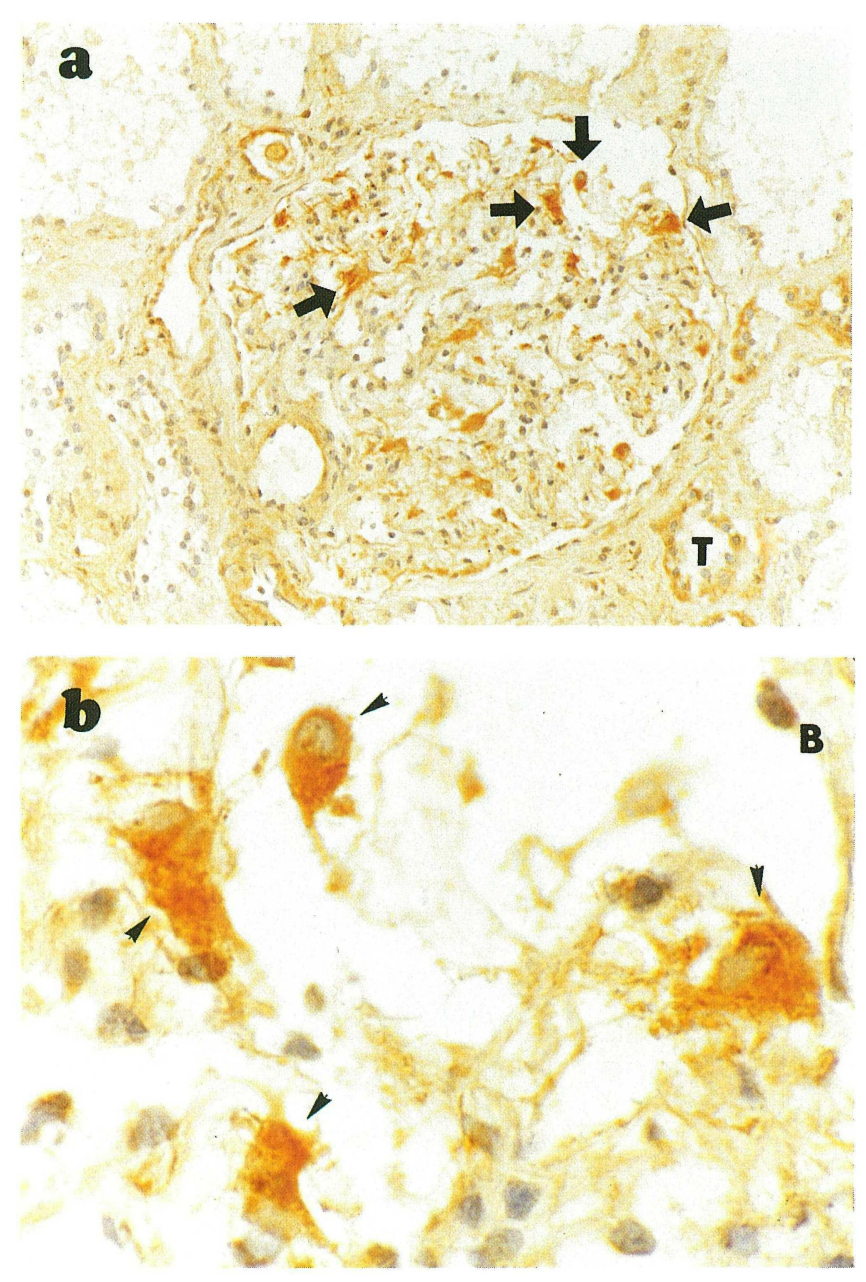

Fig. 1. In situ hybridization using a digoxigenin (DIG)-labeled Il-6 antisense oligonucleotide probe on a renal biopsy specimen from a patient with IgA nephropathy, a) a low magnification $(\times 200)$, there were strongly positive cells (arrows) in the glomeruli. b) a higher magnification of glomerulus $(\times 1,000)$; a positive signal was clearly observed in a cytoplasmic pattern (arrow-heads). In negative cells, only nuclei were stained by hematoxylin in negative cells. T, tubules; B, epithelial cells of Bowman's capsule.

nephropathy is shown in Fig. 1a. This patient had moderate tissue damage with mesangial cell proliferation and mesangial matrix expansion, as shown by periodic-acid Schiff staining. IL-6 mRNA positive cells were clearly demonstrated in glomeruli. At a higher magnification (Fig. 1b), a clear distinctive signal is apparent in the cytoplasm of positive cells. Negative cells were only detectable by hematoxylin counterstaining of the nuclei. The low level of background staining allows this clear distinction. A variety of controls to verify signal specificity were performed. Treatment of tissue sections with RNase prior to hybridization eliminated most of the signal obtained with the IL-6 probe (Fig. 2, a and b). As an alternative approach, when a 100-fold excess of unlabeled IL-6 anti-sense oligonucleotide was added to the standard hybridization mixture containing DIG-labeled IL-6 probe, the positive signal was completely abolished (Fig. 1c). On the other hand, in the presence of a 100-fold excess amount of a non-homologous unlabeled oligonucleotide (B-actin), the signal of IL-6 mRNA was not effective (Fig. 1d). In addition, a DIG-labeled IL-6 sense probe did not yield any positive signals (Fig. 2, e and f). For physiological controls, LPS-stimulated or unstimulated PBMC were examined. LPS-stimulated cells exhibited a strong hybridization signal with the IL-6 antisense probe, while unstimulated PBMC were almost all negative (data not shown).

\section{Discussion}

The development of a sensitive method of non-radioactive in situ hybridization was dependent upon a number of factors. First, the estimation of digoxigenin incorporation into the oligonucleotide probe was critical. This was performed by spotting $1 \mu \mathrm{l}$ aliquots of serial 10 -fold dilutions of the probe and detection of DIG-labeled material by the 4-layer immunoperoxidase system. It was found that when $1 \mathrm{pg}$ of labeled oligonucleotide could be clearly detected, this gave an optimal hybridization signal. A signal was obtained from probes detectable at $10 \mathrm{pg}$, but the result was weaker. Hence, each batch of labeled probe must be checked by this procedure. Secondly, deproteinization of tissue sections enhanced the hybridization signal, as has been shown in other studies of in situ hybridization $(6,7)$. This is thought to work by degrading three-dimensional protein laticeworks and removing protein molecules bound to mRNA, thus improving access of the probe to the target sequence. Determination of the optimal conditions for this step appears to be variable for each application of in situ hybridization. Thirdly, it was found that higher concentrations of DIG-labeled probes were required to obtain an optimal signal compared to that required for radiolabeled probes $(1 \mathrm{ng} / \mu \mathrm{l}$ compared to $0.15 \mathrm{ng} / \mu \mathrm{l}$ respectively). This is consistent with the difference in concentration of DIG-labeled and radiolabeled probes used in Northern blotting. It seems that the highest possible efficiency of hybridization with non-radioactive probes is necessary to allow detection, while this is less critical for radiolabeled probes. Finally, the use of the 4-layer immunoperoxidase system for the detection of the hybridization probe produced a very considerable increase in the sensitivity of signal detection. Thus, increasing the number of peroxidase molecules deposited on the section enhanced the sensitivity of detection. In addition, the use of multiple probes may be of significant benefit in the detection of low abundance mRNA species.

The sequence specificity of this method of in situ hybridization was checked by a variety of means. The ability of excess unlabeled IL- 6 probe, but not excess $B$-actin probe, to inhibit hybridization by DIG-labeled IL-6 probe was clear cut. Also, the use of an IL-6 probe of a complementary sense sequence gave no hybridization signal. However, the use of sense probes as controls must be done with caution, since it has been reported that anti-sense mRNA for some molecules does exist and can be detected by sense probes $(8,9)$. RNase treatment markedly reduced the hybridization signal, which meant that the signal was yielded by mRNA. Although RNase treatment sometimes 

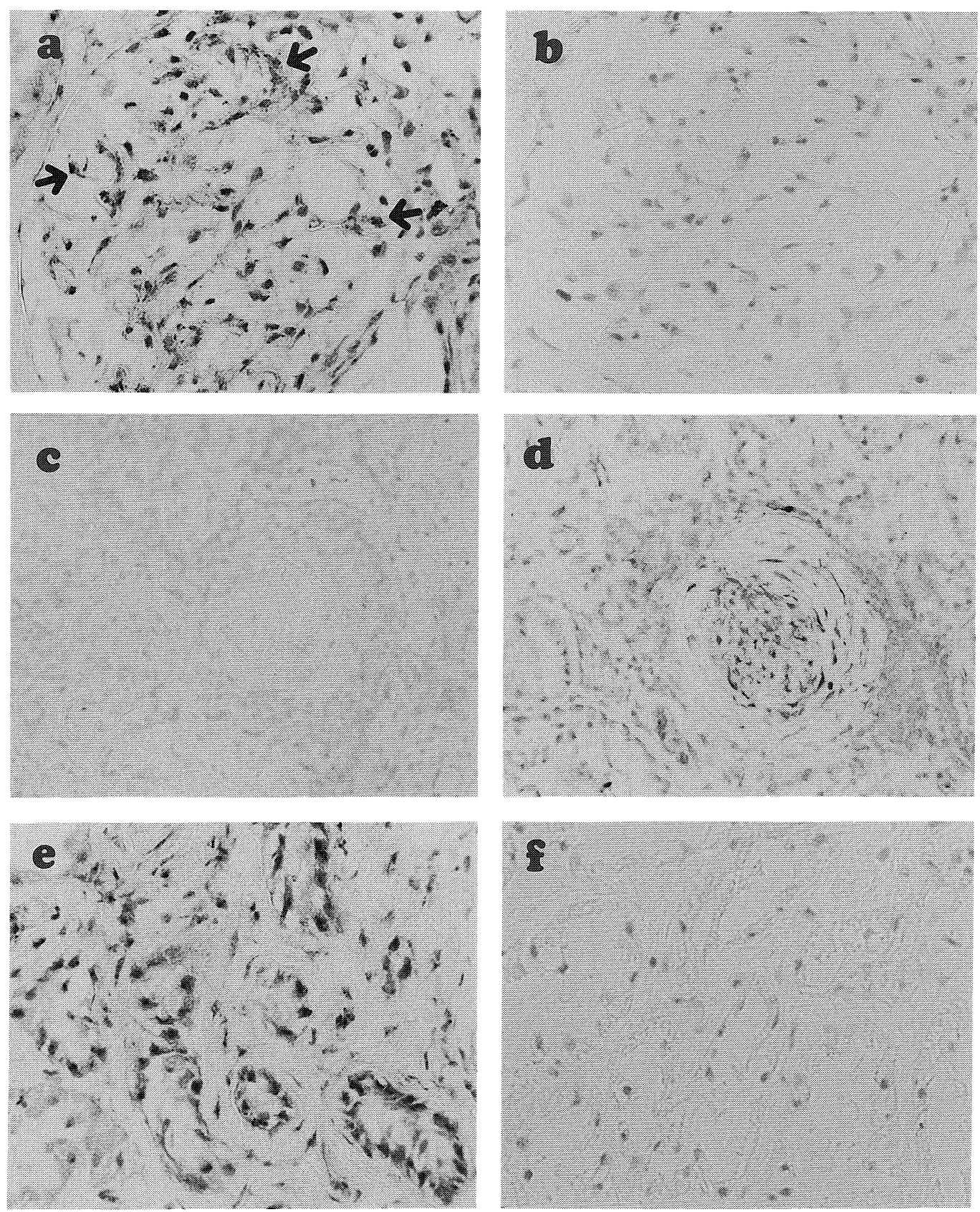

Fig. 2. Various controls for specificity. The sections used in Figs. 2-a, c and e were adjacent sections to those of Figs. 2-b, d and f, respectively. a) a section was hybridized with IL-6 antisense probe. Many positive cells were seen in glomerulus (arrows). b) an adjacent section was treated with RNase prior to hybridization. Only nuclei were seen by hematoxylin staining. c) a section was hybridized with IL-6 antisense probe in the presence of 100-fold excess amount of unlabeled IL-6 homologous oligonucleotide. The positive signals were eliminated. d) an adjacent section was hybridized with IL- 6 antisense probe with a 100 -fold excess amount of unlabeled B-actin oligonucleotide. The signal for IL-6 probe was not altered by non-homologous unlabeled B-actin oligonucleotide. e) a section was hybridized with IL-6 antisense probe. Tubular epithelial cells were stained clearly. f) hybridization with IL- 6 sense probe did not produce any positive signals $(\times 400)$.

does not entirely abrogate the hybridization signal, this is probably due to crosslinking produced in the fixation step not allowing full access of the enzyme to mRNA and the retention of cleaved fragment of mRNA. Longer incubation times and increased concentrations of RNase were also used, but a marked loss of morphology resulted. LPS-stimulated and unstimulated PBMC were examined as physiological controls using an IL-6 probe since LPS-stimulated PBMC were reported to produce mRNA of IL-6 (10). The differential hybridization of the IL-6 probe to LPS-stimulated and unstimulated PBMC provides a 


\section{In Situ Hybridization Using Digoxigenin}

simple and effective control system to ensure that the technique is working.

In conclusion, in situ hybridization using DIG-labeled oligonucleotides combined with the 4-layer immunoperoxidase staining method provides a sensitive technique for the detection of specific mRNA species within individual cells. This method is expected to be a powerful technique for molecular biological study of human renal biopsy specimens.

Acknowledgments: A part of this study was supported by grants from the Ministry of Health and Welfare of Japan. I thank to Izumi Miyazaki for assistance and preparation of the manuscript.

\section{References}

1) Arnheim N, T White, Rainey WE. The application of PCR: Organismal and population biology. Bio Science 40: 174, 1990.

2) Eisenstein B. The polymerase chain reaction: a new method of using molecular genetics for medical diagnosis. N Engl J Med 322: 178, 1990.

3) Unger ER, Hammer ML, Chenggis ML. Comparison of ${ }^{35} \mathrm{~S}$ and biotin as labels for in situ hybridization: Use of an HPV model system. J Histochem
Cytochem 39: 145, 1991.

4) Hirano $T$, Yasukawa $K$, Harada $H$, Taga $T$, et al. Complementary DNA for a novel human interleukin (BSF-2) that induces B lymphocytes to produce immunoglobulin. Nature 324: 73, 1986.

5) Ponte P, NG-SY, Engel J, Gunning P, Kedes L. Evolutionary conservation in the untranslated regions of actin mRNAs. Nucleic Acid Res 12: 1687,1984

6) Lewis ME, Sherman TG, Burke S, et al. Detection of proopiomelanocortin mRNA by in situ hybridization with an oligonucleotide probe. Proc Natl Acad USA 83: 5419, 1986.

7) Koji T, Moriuchi T, Nakane PK. Improved tissue preparation for in situ localization of specific mRNA using non-radioactive DNA probes: Effects of protease digestion and probe size on signal detection in frozen and paraffin sections of rat pituitary glands. Acta Histochem Cytochem 21: 187, 1988.

8) Kimelman D, Kirschner MW. An antisense mRNA directs the covalent modification of the transcript encoding fibroblast growth factor in Xenopus oocytes. Cell 59: 687, 1989.

9) Roberston NG, Pomponio RJ, Mutter GL, Morton CC. Testis-specific expression of the human MYCL2 gene. Nucleic Acids Res 19: 3129, 1991.

10) van Snick J. Interleukin-6: an overview. Ann Rev Immunol 8: 253, 1990. 